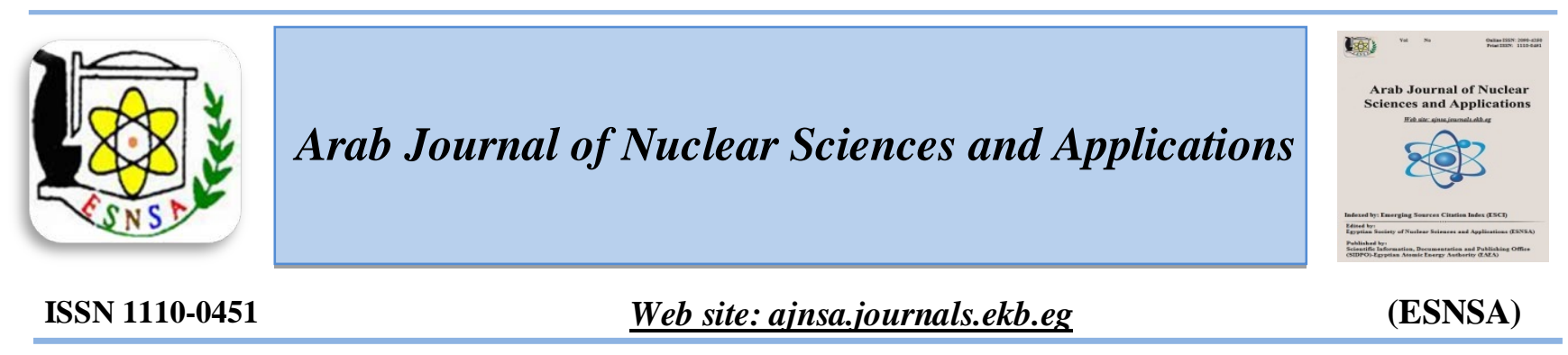

\title{
Self-Calibrating Enabled Low Cost, Two Channel Type K Thermocouple Interface for Microcontrollers
}

\author{
H. H. Shaker, A.A. Saleh, A. H. Ali, and M. Abd Elaziz \\ Egypt Second Research Reactor Complex, Atomic Energy Authority (AEA)
}

\begin{abstract}
Received $1^{\text {st }}$ Sept 2016 This paper presents a new design of a very low cost two-channel type $K$ thermocouple interface circuit Accepted $10^{\text {th }}$ Oct 2016 for micro-controllers. This interface circuit targets accurate temperature measurements for nuclear research instrumentation from 0 up to 250 degree Celsius with 1 degree resolution. The usage of the computational capabilities of a micro-controller is proposed to make an adaptive self calibration for the designed interface circuit. Also, these computational capabilities are used to solve the two major problems associated with thermocouples, namely the thermocouple non-linearity problems and the offset errors resulting from the thermocouple reference junctions.
\end{abstract}

Keywords: Thermocouple amplifier board, self-calibration, microcontroller

\section{Introduction}

A thermocouple is a sensor used to measure temperature. It consists of two twisted wires made of different metals. The wires terminals are welded together at one end, creating a junction. The temperature is measured at this junction. When the junction experiences a change in temperature, a voltage is created. There are many types of thermocouples, each with its own unique characteristics in terms of temperature range, durability, vibration resistance, chemical resistance, and application compatibility. Types J, K, T, \& E are base metal thermocouples, which are the most common types. Types R, S, and B are noble metal thermocouples, which are used in high temperature applications [1].

Thermocouples are used in many fields of the nuclear industry, and research. They are typically selected due to their low cost, high temperature limits, and wide temperature ranges. They are a good choice for flexible temperature measurement because they can be thousands of feet long without affecting the temperature measurement accuracy [2].

An interface circuit is needed between the thermocouple terminals and the microcontroller analog input pins. This interface makes it possible for the microcontroller to accurately understand the output of the thermocouple signal. A lot of single channel thermocouple interface (amplifier) boards are available in the electronics market at a price ranging from $11 \$$ to $22 \$$ (as in ebay shop). Their cost is not low because all the thermocouple measurement difficulties are locally solved in the interface board before its connection to the microcontroller.

In the present work, a design of a very low cost $(<3 \$)$ two channel thermocouple interface circuit is proposed. This design is based on the operational amplifiers implemented in the LM358 integrated circuit ( $0.05 \$$ in ebay shop). This interface targets accurate temperature measurements for nuclear research instrumentation from 0 up to 250 degree Celsius with 1 degree resolution. The temperature measurement accuracy is guaranteed

Corresponding author: H. H. Shaker

DOI: 10.21608/ajnsa.2018.12391

(c) Scientific Information, Documentation and Publishing Office (SIDPO)-EAEA 
by proposing the use of the microcontroller computational capabilities. This allows making an adaptive self-calibration for the designed interface circuit. These computational capabilities are also used to solve two major problems associated with thermocouples: the thermocouple non-linearity problems; and the offset errors resulting from the thermocouple reference junctions.

Arduino is an open source microcontroller-based kit. Such kits are flexible and easy to use, and they provide sets of digital and analog input/outpot ports that can be connected to other expansion boards to build up a complex embedded systems. Arduino boards are produced by several vendors, using different microcontrollers [3]. Arduino-Uno is based on Atmega328P microcontroller, and it is used in this work as a microcontroller example to validate the designed interface circuit.

The detailed block diagram of the proposed interface board is clarified in section II with a brief explanation. Section III presents the design of the differential amplifiers which are the main building blocks of the interface circuit. Section IV discusses the problem of the thermocouple reference junction and clarifies the implemented solution. Section V discusses the importance of using an adaptive selfcalibration technique to compensate any variation in the amplifiers parameters. The hardware and software descriptions are included in sections VI, and VII respectively. Finally, the validating experimental results and conclusions are presented in section VIII and IX.

\section{The Proposed Interface Circuit Block Diagram}

Figure (1) presents the detailed block diagram of the proposed thermocouple interface circuit. Two thermocouples can be connected as inputs to this circuit and their conditioned outputs present on AI1, and AI2 pins, which have to be connected to the analog input pins of the microcontroller.

- The fixed offset batteries are used to shift up the thermocouple signal: this helps avoiding the lower non-linear region of the differential amplifiers; and this allows a temperature measurement down to 0 degree Celsius.

- $\quad$ The divider blocks receive a 0 to $5 \mathrm{~V}$ ramp DC voltage signal from a microcontroller's analog output $\mathrm{AO}$ pin and divides it by a certain factor to provide a ramp signal that simulates the thermocouple output signal which its measurement junction is attached to a surface with an increasing temperature.

- The switching network is responsible for switching the input ports of the differential amplifiers between the actual and the simulated thermocouple signals. In other worlds, the switching network is responsible for switching between the normal and the self-calibration modes.

- The differential amplifiers multiply the thermocouple signals by a large gain to be easily measured by the analog to digital converter ADC included in the microcontroller.

- The low pass filter LPF blocks rejects out any noise down to $50 / 60 \mathrm{~Hz}$ (may be introduced from the mains supply).

- Finally, the temperature sensor is included to compensate the offset errors resulting from the thermocouple connection junctions, and it is also used as an indicator that a new self -calibration is needed

\section{Differential Amplifier Design}

LM358 is a very cheap dual operational amplifier integrated circuit IC. It consists of two independent operational amplifiers Op-Amps A, and B. These Op-Amps can operate with a single power supply, and their input common mode can be in the ground level [4]. These features allow the use of the same supply of the microcontroller for energizing the interface circuit. Additionally, the thermocouple signals can be applied directly to the input of the differential amplifiers without any common mode voltage level. This facilitates designing a large DC gain by a single stage amplifier. Atmega328P micro-controller includes 10 bit ADC [5] which gets its reference voltage by default as same as the regulated supply voltage of the Arduino board (5 V ideally). This means that the least significant bit of this ADC is equivalent to $4.9 \mathrm{mV}$. On the other hand, type $\mathrm{K}$ thermocouple signal changes approximately by $40 \mathrm{uV}$ per 1 degree C [6]. A resolution of 1 degree $\mathrm{C}$ can be achieved by multiplying the thermocouple signal by a gain factor higher than $122(4.9 \mathrm{mV} / 40 \mathrm{uV})$. The OpAmps of LM358 IC are used to implement two differential amplifiers with a gain factor equivalent to 200. The differential topology is used to suppress any common mode noise super-imposing on the 
thermocouple signal or on the ground level. Two experimental test benches were conducted for these differential amplifiers. The first test bench extracts the transfer functions for three different LM358 ICs. As shown in Figure (2), all amplifiers have the same gain factor (the same slope), but each one has a different offset shift (problem 1). It is better to use the pure linear range of the amplifier between $5 \mathrm{mV}$ to $16 \mathrm{mV}$. This is done by connecting the negative terminal of the thermocouple to a $5 \mathrm{mV}$ DC offset referred to the ground, and measuring the output voltage between the positive terminal of the thermocouple and ground. The second test bench determines the effect of changing the interface circuit surrounding temperature. Figure (3) show a plot for the normalized gain and offset of the differential amplifier (using IC1_A Opamp) at different surrounding temperatures. The gain changed slightly, but the offset changed dramatically (problem 2). The implemented solutions to overcome the two problems of this cheap amplifier are discussed in section V.

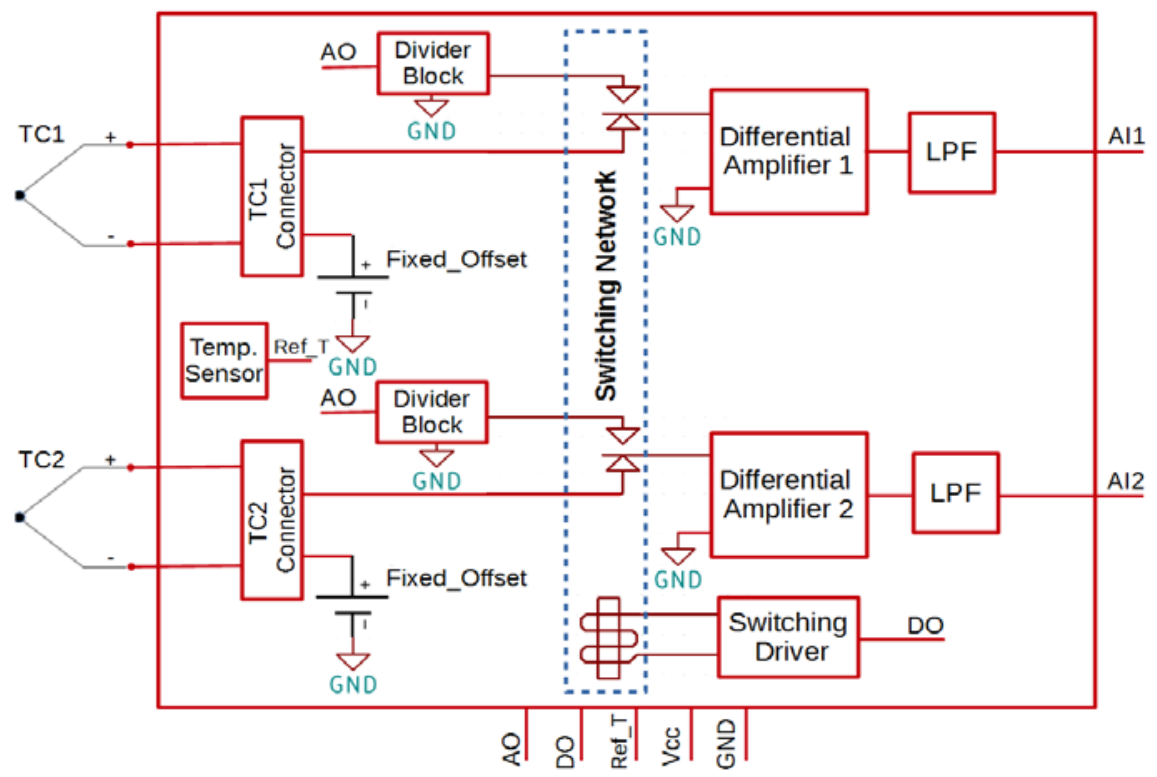

Figure (1): The proposed interface circuit block diagram

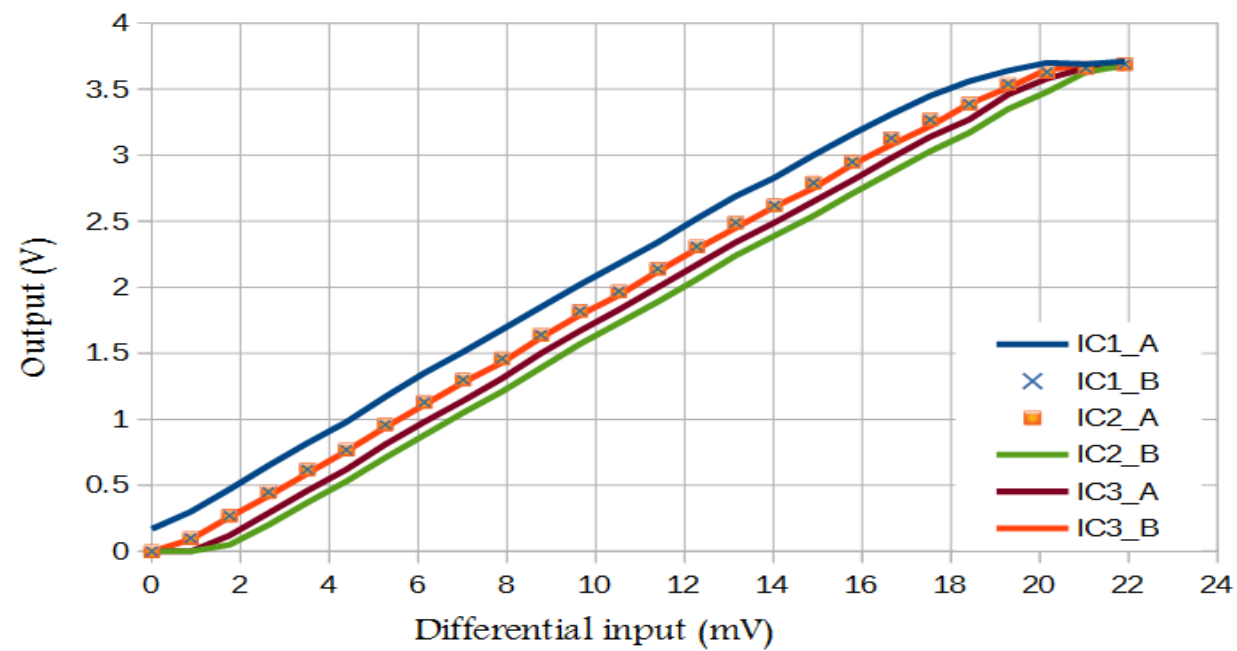

Figure (2): Transfer function of the differential amplifiers for three different IC 


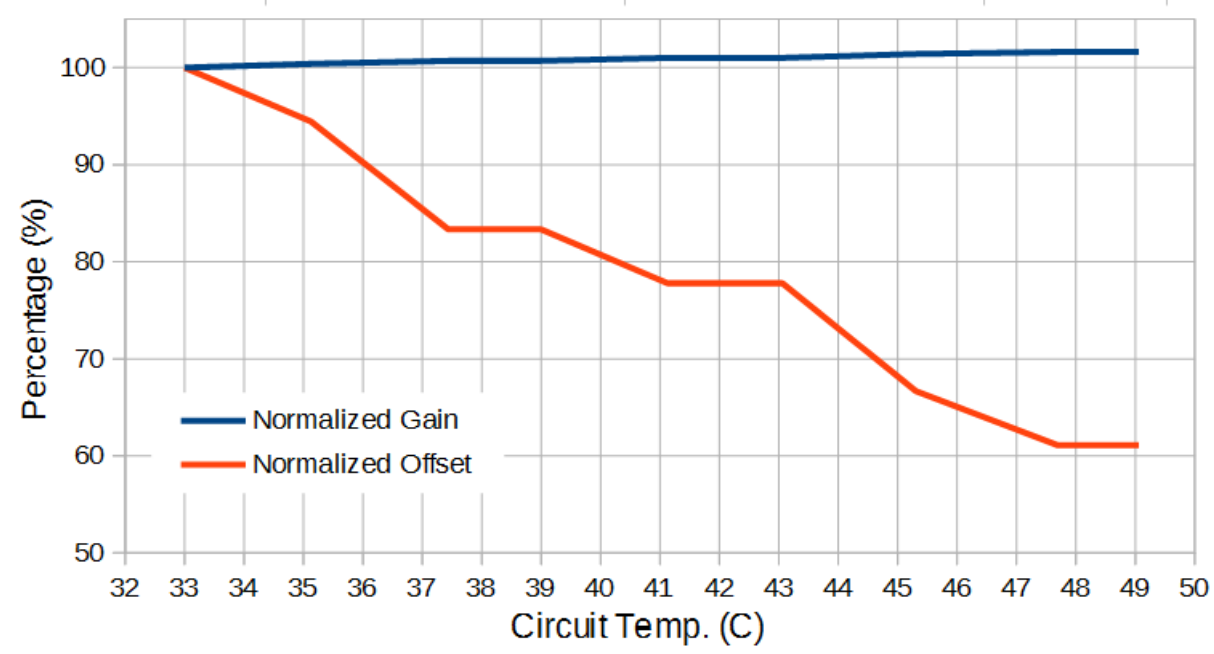

Figure (3): Normalized gain and Normalized Offset of IC1_A versus circuit temperature (C)

\section{Reference Junction Compensation}

The thermoelectric effect may appear at any connection junction of two different metals [7]. When connecting type $\mathrm{K}$ thermocouple terminals (Alumel and Chromel metals) to a PCB connector (Copper metals), two thermocouple junctions appear. If these connection junctions (called reference junctions) are at the same temperature, they will induce a negative potential drop equivalent to that induced from thermocouple type $\mathrm{K}$ when measuring temperature $\mathrm{T} 2$ (as shown in Figure 4) [7]. This potential drop should be taken into consideration in the temperature measurement calculations in the microcontroller software.

As clarified in table (I), the reference junction error can be compensated by accurately measuring the temperature $\mathrm{T} 2$, then converting this value into its equivalent induced voltage from thermocouple type $\mathrm{K}$. Finally, the microcontroller uses this voltage value as a reference for the temperature measurement calculations [7]. DS18B20 is a digital thermometer IC, which is used here to implement the temperature sensor block. It is featured by a 1wire communication capability, and it allows temperature measurements from -55 to 125 degree Celsius by $+/-0.5$ accuracy [8].

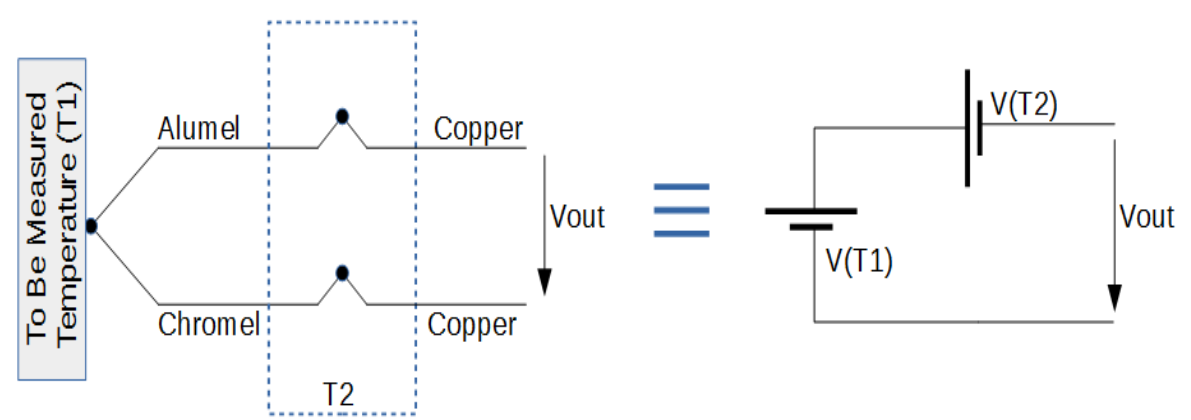

Figure (4): Equivalent circuit of the thermocouple sensing and connection junctions 
TABLE (1): Typical coding for reference error compensation

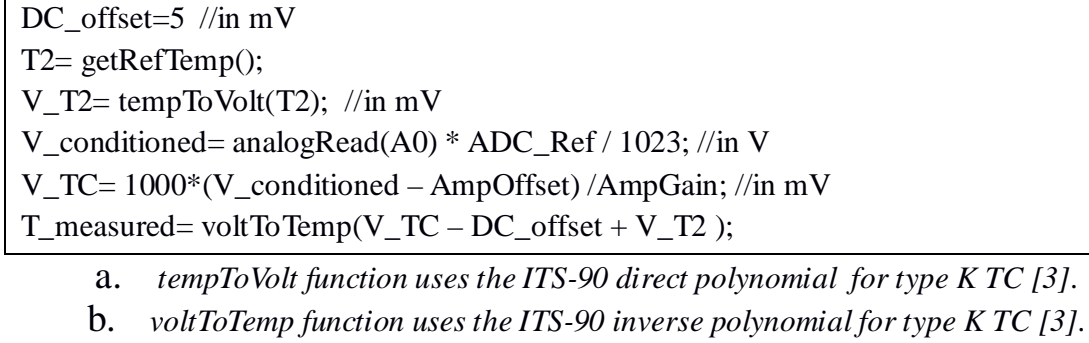

\section{Self-Calibration Technique}

The offset of the amplifier may change with the variation of the surrounding temperature as well as with changing the amplifier IC itself as was mansion in section III. The measurement accuracy can be maximized by using the self-calibration algorithm. Self-calibration should be conducted as often as necessary [9]. It can be triggered manually after changing the amplifier IC by an external push button or through communication interfaces of the microcontroller such as serial interface. It may also be performed automatically by the microcontroller when the surrounding temperature of the amplifier changes by +/-1 degree $C$ with respect to the last calibration. The self-calibration is enabled in the proposed thermocouple interface circuit by implementing the switching network which flips the amplifiers inputs to the output of the divider blocks during the self-calibration mode. The divider blocks get an input square wave with an increasing duty cycle in steps from the microcontroller analog output pin, and it extracts an attenuated DC voltage from 0 up to $22 \mathrm{mV}$ range. For each step, the amplifiers outputs are stored in a look up table. Finally, the amplifiers' gain and offset are calculated, stored, and used in the temperature measurement calculations.

\section{Hardware Description}

KiCad is open source software for electronic design automation. It facilitates the stages of the printed circuit board PCB design using an integrated environment [10]. Figure (5) presents a schematic diagram of the designed interface circuit as drawn in KiCad. This schematic diagram is passed to the layout editor included in KiCad, and a single side layout is designed. Finally, the fabrication files are extracted and delivered to the EAEA PCB fabrication lab. Figure (6) presents the fabricated sample board which was used to validate the proposed concept.

\section{Software Description}

An Arduino library is written to allow easy usage of the designed interface circuit. The library consists of three functions: TC_measure function, tempToVoltage function, and voltageToTemp function. TC_measure can be called to get the temperature values for the two thermocouples connected the the interface circuit. And it calls internally the other two functions. Figure (7) clarifies the flow chart of the TC_measure function. First, it gets the circuit temperature indication from the DS18B20 IC. If the circuit temperature is not $+/-5$ degree around the last calibration temperature, then, it starts a new self-calibration and saves the new gain and offset values for each amplifier. Finally, it begins the algorithm (as illustrated in Table. 1) for the temperature measurement for each thermo-couple and returns two pointers for the measured temperature. TempToVoltage and voltageToTemp functions use the thermocouple type $\mathrm{K}$ direct and inverse polynomials that were published in a previous study [2]. These polynomials compensate perfectly the non-linearity problems of the thermocouple.

\section{Design Validating Results}

Three experimental test benches were conducted to validate the designed interface board: the first test bench uses a mercury-in-glass thermometer as a trusted temperature measurement tool to validate the measured temperature values using the new design. The second test bench uses a commercial temperature controller to guarantee the ability of the new design to measure temperatures up to 250 degree $\mathrm{C}$. The third test bench guarantees the temperature measurement accuracy at different interface circuit surrounding temperature values. During the test benches, the interface circuit is connected to the Arduino board which is connected to a laptop through USB cable. The previously written library is uploaded to the Arduino, and the measured temperature values are sent and displayed on the laptop monitor using the serial monitor software (included in Arduino environment). 


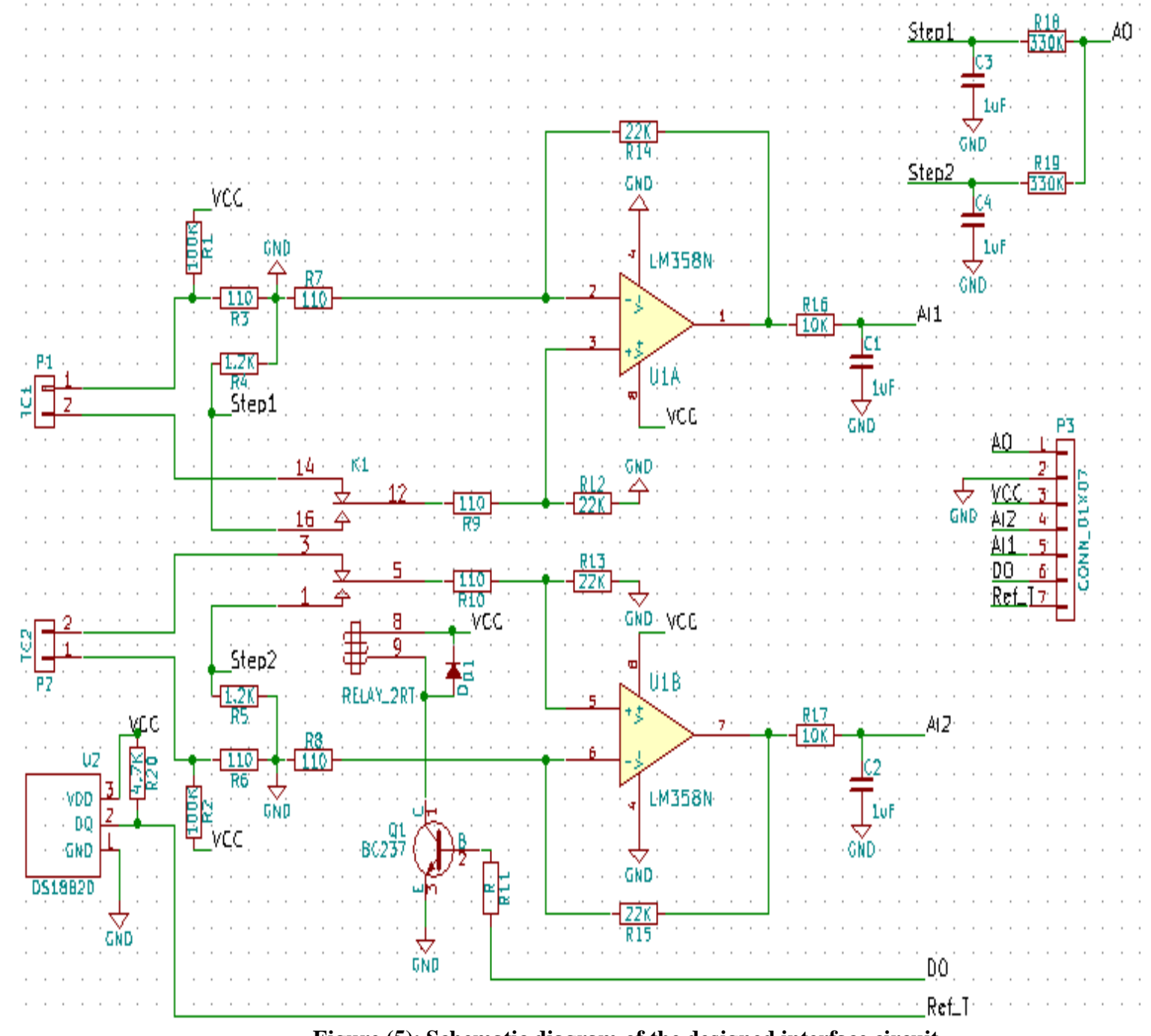

Figure (5): Schematic diagram of the designed interface circuit

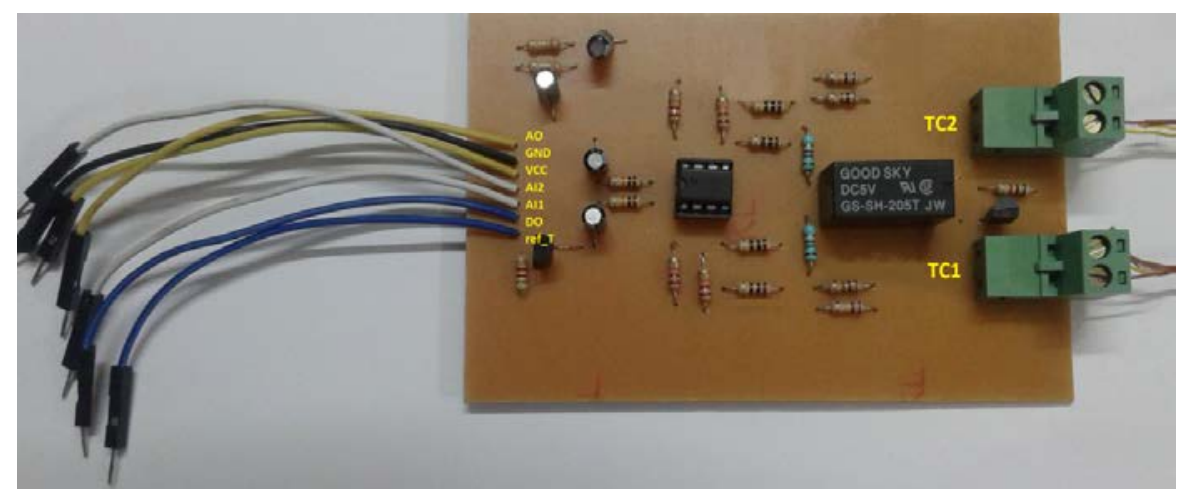

Figure (6): The fabricated PCB of the designed interface circuit (first version) 


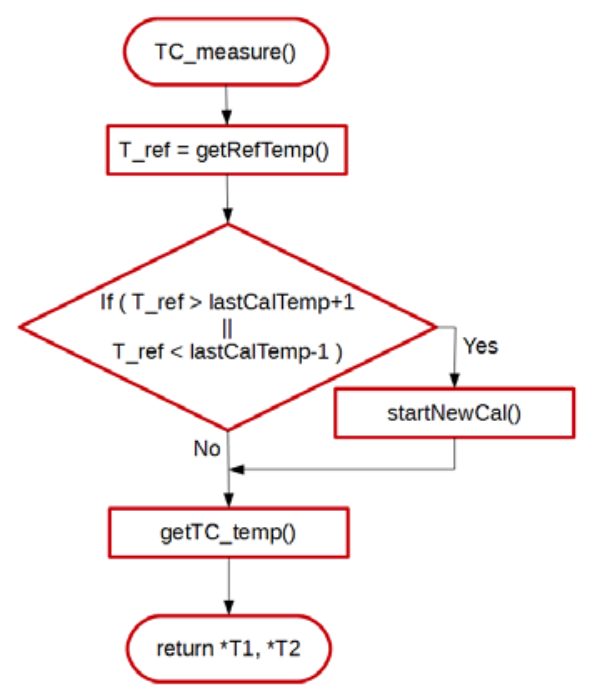

Figure (7): Flow chart of TC_measure function

\section{A. First test bench}

Two thermocouples were connected to the designed interface circuit. The measurement junctions of the two thermocouples are attached to the sensing bulb of a mercury-in-glass thermometer which was inserted in a pool of water. The water was heated up to the boiling point then it was cooled in steps. Figure (8) plots the experimental results. The temperature measured by the designed interface circuit achieved a very good matching with respect to that measured by the glass thermometer.

\section{B. Second test bench}

TC4Y-14R is a temperature controller which is manufactured by Autonics. It accepts signals from a resistance temperature detector, or from a thermocouple and displays the temperature value on a four digit 7 segment display [11]. Three thermocouples are used to setup this experimental test bench. One thermocouple is connected to the TC4Y controller, and the other two are connected to the designed interface circuit. The measurement junctions of the three thermocouples are attached together to a heater, the heater is warmed until reaching 250 degree $\mathrm{C}$, and then it is unplugged from the power source. The temperature decay of the heater is monitored every 10 seconds by the three attached thermocouples. Figure (9) shows a plot for the experimental results. The designed interface circuit achieved a very good temperature measurement with respect to that measured by the TC4Y controller.

\section{Third test bench}

One thermocouple was connected to the designed interface circuit. The measurement junction of the thermocouple was inserted in a pool of water. The interface circuit is fixed close to an oven. The oven was operated, which resulted in increasing the surrounding temperature of the interface circuit. This experiment is conducted two times: in the first time, self calibration is operated at the start of the experiment and at 1 degree $\mathrm{C}$ change of the surrounding temperature of the interface circuit; in the second time, self calibration is only operated at the start of the experiment. Figure (10) shows a plot for the experimental results. It is shown clearly that self-recalibration feature in the proposed design guarantees very good measurement accuracy at different interface circuit surrounding temperature values. 


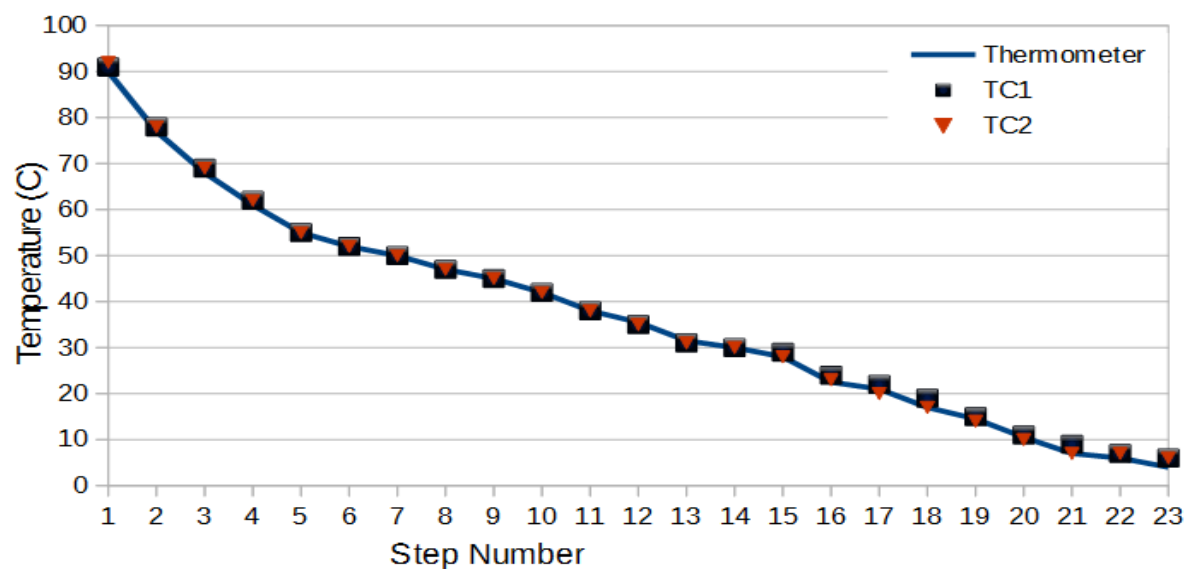

Figure (8): Measured Temperature versus cooling step number from the thermometer and the two thermocouples which are connected to the designed interface circuit.

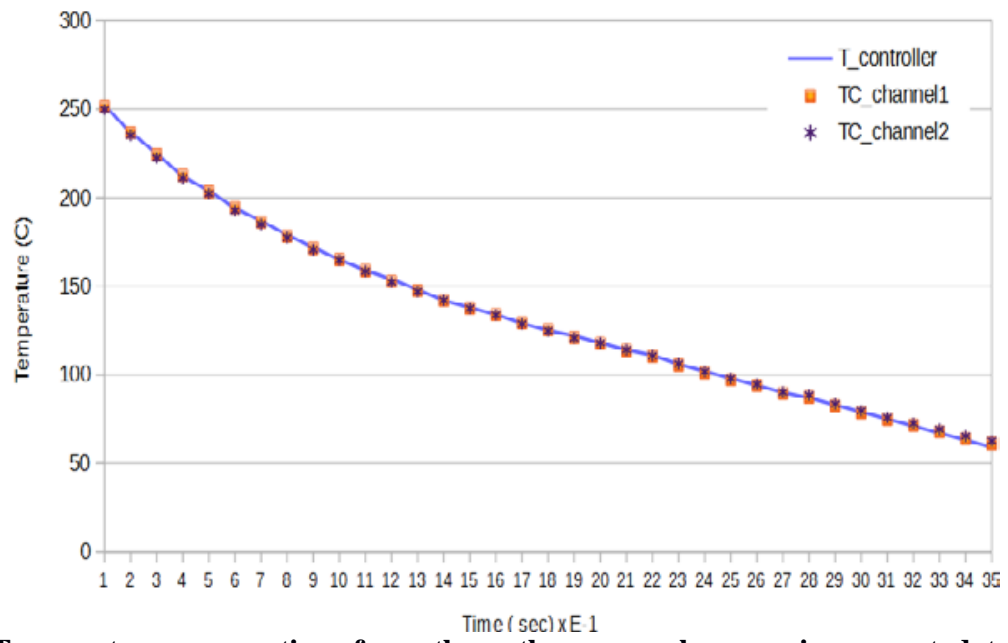

Figure (9): Measured Temperature versus time from three thermocouples, one is connected to a temperature controller as a reference, and the other two are connected to the designed interface circuit.

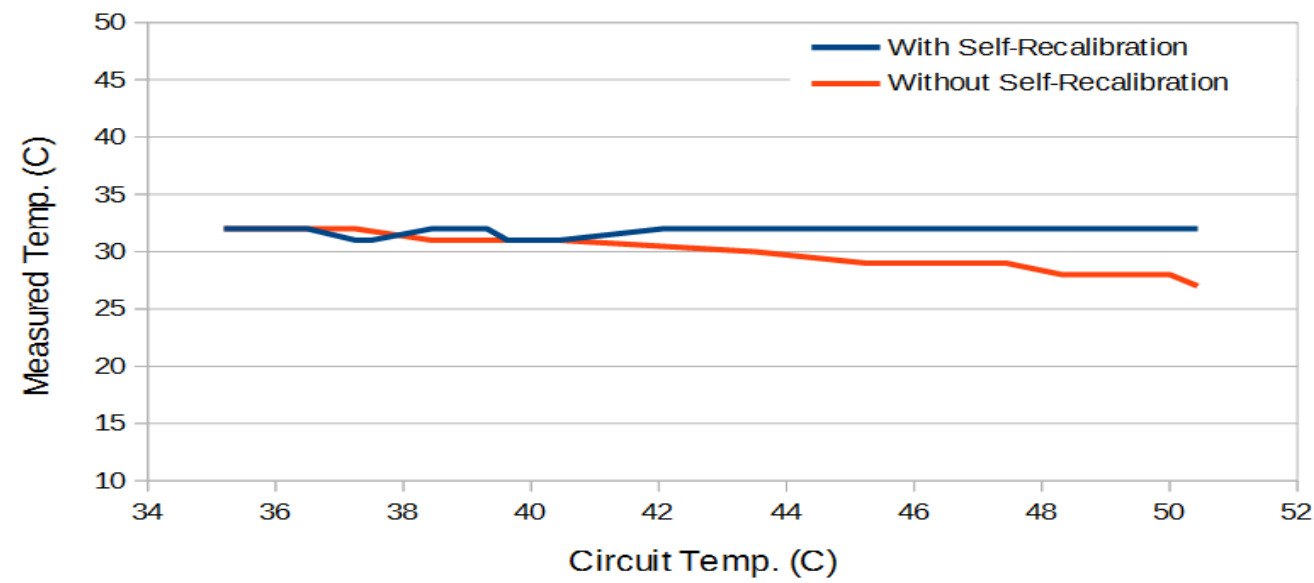

Figure (10): Measured temperature versus interface circuit surrounding temperature with enable and disable of selfrecalibration 


\section{Conclusions}

The present research proposed a design of a very low cost two channel thermocouple interface circuit, based on the operational amplifiers implemented in the LM358 IC. This interface targets an accurate temperature measurement for nuclear research instrumentation from 0 up to 250 degree Celsius with 1 degree resolution. The temperature measurement accuracy was guaranteed by proposing the use of the microcontroller computational capabilities. This allowed making an adaptive self calibration for the designed interface circuit as well as solving the two major problems associated with thermocouples, namely the thermocouple non-linearity problems; and the offset errors resulting from the thermocouple reference junctions. The proposed interface circuit is designed, fabricated, and validated.

\section{References}

1- "Thermocouple-Thermocouples-What is a thermocouple-Types of thermocouples", Thermocoupleinfo.com, 2016. [Online]. Available: http://www.thermocoupleinfo.com/. [Accessed: 07Aug- 2016].
2- R. Bentley, Handbook of Temperature Measurements, 1998, Sec. Z.

3- "Arduino", Wikipedia, 2016. [Online]. Available: https://en.wikipedia.-org/wiki/Arduino. [Accessed: 07- Aug- 2016].

4- ST Microelectronics, "Low power dual operational amplifiers”, LM358 datasheet, January 2002.

5- ATMEL, "High performance, low power AVR 8bit microcontroller", Atmega328P datasheet, Rev. 8161C.

6- M. Duff, and J. Towey, "Two ways to measure temperature using thermocouples feature simplicity, accuracy, and flexibility”, Analog Dialogue, vol 44, October 2010.

7- W. Kester, J. Bryant, and W. Jung, Practical Design Techniques for Sensor Signal Conditioning, Analog Devices, 1999.

8- DALLAS Semiconductor, "Programmable resolution 1-wire digital thermometer”, DS18B20 datasheet.

9- VTI Instruments, How to Maximize Temperature Measurement Accuracy, Appl. Note.

10- "KiCad", Wikipedia, 2016. [Online]. Available: https://en.wikipedia.org/wiki/KiCad. [Accessed: 07- Aug- 2016].

11- Aotunics, “Temperature controller TC4 series", TC4Y datasheet. 